\title{
Desregulación emocional y conducta disocial en una muestra de adolescentes en conflicto con la ley ${ }^{1}$
}

\author{
Germán Cabrera Gutiérrez², Nora Helena Londoño Arredondo³, Joan Sebastián Arbeláez Caro4, \\ Juan David Cruz Valencia ${ }^{5}$, Leidy Yovanna Macías Castillo ${ }^{6}$, Ana María España Macías \\ Universidad de San Buenaventura Medellín, Armenia (Colombia)
}

Recibido: 06/07/2019 Aceptado: 26/06/2020

\begin{abstract}
Resumen
Objetivo. Establecer el grado de relación entre la desregulación emocional y la conducta antisocial y delictiva en adolescentes que se encuentran en conflicto con la ley. Método. Diseño no experimental, enfoque cuantitativo, con alcance descriptivo-correlacional de temporalidad transversal. Participaron 62 adolescentes en conflicto con la ley. Se implementaron la ficha de caracterización MINI KID y la Escala de Desregulación Emocional (DERS-E). Resultados. El 54.8\% de los adolescentes reportaron problemas de conducta. Según los hallazgos, la desatención emocional es diferente entre las personas con trastorno disocial y las que no lo presentan $(t=4.853, p=0.031)$. La desatención emocional predice la aparición de trastorno disocial $(\beta=0.030$, $p=0.05, \operatorname{Exp}(\beta)=1.362)$. Conclusión. Los datos dan cuenta de la importancia de la revisión teórica de la conducta antisocial y de los factores asociados a ella, para establecer mejores modelos de intervención y de comprensión del fenómeno, principalmente la influencia de la regulación emocional en la aparición de la conducta disocial en adolescentes.
\end{abstract}

Palabras clave. Regulación emocional, desórdenes comportamentales, conducta delictiva, conducta adolescente.

\section{Emotional Dysregulation and Dissocial Behavior in a Sample of Adolescents in Conflict with the Law}

\footnotetext{
Abstract

Objective. To establish the degree of relationship between emotional dysregulation and antisocial and criminal behavior in adolescents who conflict with the law. Method. Non-experimental design, quantitative approach,

Artículo de investigación realizado en el marco del proyecto "Desenganche moral, desregulación emocional y prácticas de crianza parental, en adolescentes vinculados y no vinculados al sistema de responsabilidad penal" de la Universidad de San Buenaventura Medellín.

2 PhD en Psicología. Docente investigador de la Universidad de San Buenaventura Medellín extensión Armenia. Correo de correspondencia: german. cabrera@usbmed.edu.co

3 PhD en Psicología. Docente investigadora de la Universidad de San Buenaventura Medellín.

4 Psicólogo. Maestrante en Enseñanza de las Ciencias. Docente investigador Universidad de San Buenaventura Medellín extensión Armenia.

5 Psicólogo.

6 Psicóloga.

7 Psicóloga.
} 
104 GERMÁN CABRERA GUTIÉRREZ, NORA HELENA LONDOÑO ARREDONDO, JOAN SEBASTIÁN ARBELÁEZ CARO, JUAN DAVID CRUZ VALENCIA, LEIDY YOVANNA MACÍAS CASTILLO Y ANA MARÍA ESPAÑA MACÍAS

with descriptive scope - correlation of transversal temporality. Sixty-two adolescents in conflict with the law participated. The instruments used were the Characterization Sheet, MINI KID and Emotional Deregulation Scale (DERS-E). Results. 54.8\% of adolescents reported behavioral problems. Emotional neglect is different between people with antisocial personality disorder and those who do not have it $(t=4.853, p=0.031)$. Emotional neglect predicts the onset of antisocial personality disorder $(\beta=0.030, p=0.05, \operatorname{Exp}(\beta)=1.362)$. Conclusion. The data showed the importance of the theoretical review of antisocial behavior and the factors associated with it in order to establish better models of intervention and understanding of the phenomenon, mainly the influence of emotional regulation on the onset of dissocial behaviour in adolescents.

Keywords. Emotional dysregulation, dissocial behavior, criminal behavior, adolescent behavior.

\section{Desregulação emocional e conduta dissocial numa mostra de adolescentes em conflito com a lei}

\section{Resumo}

Escopo. Estabelecer o grau de relação entre a desregulação emocional e a conduta antissocial e delitiva em adolescentes que estão em conflito com a lei. Metodologia. Desenho não experimental, enfoque quantitativo, com alcance descritivo-correlacional de temporalidade transversal. Participaram 62 adolescentes em conflito com a lei. Foi implementada a ficha de caracterização MINI KID e a escada de Desregulação Emocional (DERS-E). Resultados. O 54.8\% dos adolescentes reportaram problemas de conduta. Segundo os achados, a desatenção emocional é diferente entre as pessoas com transtorno dissocial e as que não a presentam $(t=4.853, p=0.031)$. A desatenção emocional prediz a aparição de transtorno dissocial $(\beta=0.030, p=0.05$, $\operatorname{Exp}(\beta)=1.362$ ). Conclusão. Os dados dão conta da importância da revisão teórica da conduta antissocial e dos fatores associados a ela, para estabelecer melhores modelos de intervenção e de compreensão do fenómeno, principalmente a influencia da regulação emocional na aparição da conduta dissocial em adolescentes.

Palavras-chave. Desregulação emocional, conduta dissocial, conduta delitiva, conduta adolescente.

\section{Introducción}

Una de las problemáticas que más interesa a la psicología como disciplina científica, es entender el comportamiento de las personas que van en contra del orden establecido. Este fenómeno se ha conceptualizado y ha evolucionado con el refinamiento de las teorías y los métodos de investigación en ciencias sociales y humanas. En psicología, particularmente, el constructo de comportamiento antisocial y delictivo no es nuevo dentro de la investigación, y cada vez son más los aportes acerca de su origen y sus efectos. La presente investigación aborda este constructo por cuanto interesa a la psicología, dada su connotación e implicaciones en la sociedad actual. El término de conducta antisocial, en sí mismo, puede resultar polisémico dadas sus relaciones en cada campo de estudio, aunque específicamente en psicología se refiere a un conjunto de conductas que impactan el bienestar subjetivo y social. Además, este es un amplio campo de investigación, concebido como el espectro de comportamientos que atentan contra la armonía que la norma social provee (López, 2008).

El comportamiento antisocial de los individuos dentro de una sociedad debe estudiarse de forma conjunta con el sistema social y familiar, teniendo en cuenta variables, aspectos y factores de los dominios biológico, cognitivo y psicosocial. En la integración de los dominios biológico y cognitivo, el abordaje desde las neurociencias modernas ha permitido comprender la conducta antisocial como parte del funcionamiento ejecutivo, así lo expresan Aldrete-Cortez, Carrillo-Mora, MansillaOlivares, Schnnas y Esquivel (2014). Estos autores han concluido que la autorregulación es un proceso que hunde sus raíces en la estructuración biológica propia de la gestación, que se continúa, además, en lo que ellos Ilaman regulación autonómica. Esta 
variable es precursora de la regulación emocional, ya que implica funciones inhibitorias, y sobre este proceso se da el sólido fundamento de las funciones ejecutivas que enmarca el proceso biológico de la regulación emocional.

Siguiendo estos aportes acerca de las teorías psicológicas que explican el comportamiento antisocial, la comprensión del desarrollo del sujeto desde la epigénesis ha dado grandes frutos, al punto que es el hilo conductor que une las teorías sobre el comportamiento humano. Hoy es ampliamente aceptado por las ciencias sociales y humanas, considerar el enfoque epigenético como el paradigma más adecuado para comprender la génesis o etiología del comportamiento antisocial y las demás formas de comportamiento, ya que gracias a él es posible sumar tanto los factores genético-estructurales con los elementos medioambientales. De hecho, Carranza (1994) define este enfoque como el "proceso de continua interacción dialéctica o coacción entre el genoma, el organismo y su ambiente" (p. 107). En esa dirección, el comportamiento e identidad del sujeto, como elementos constitutivos de la personalidad, son la expresión de la modulación que se da entre las cargas biológicas constitutivas de la persona y su ambiente natural, el cual incluye las instituciones creadas por la sociedad humana como la familia y el lenguaje.

Sin embargo, la teoría epigenética resuelve una parte de las consideraciones etiológicas del comportamiento antisocial, ya que comprender en qué grado y en qué medida se conjugan las características biopsicosociales es una labor más amplia y corresponde a la investigación desde la disciplina psicológica. De esta manera, la evidencia apunta a que la regulación emocional está implicada en la conformación del comportamiento antisocial. Al estudiar la dependencia entre la desregulación emocional y la conducta antisocial, Gutiérrez, Herrero y Zarabia (2014) hallaron que la primera variable funciona como un factor que podría evidenciar que el sujeto desarrolle conductas antisociales. El impacto que podría tener una adecuada ordenación emocional o una adecuada habilidad emocional (Gutiérrez et al., 2014) indica el grado de desorden intrapsíquico. De hecho, la importancia del estudio del control de las emociones tiene que ver con la idea de que es un predictor de la conducta antisocial (Garaigordobil et al., 2013).
Desde el dominio psicosocial y cognitivo, Gross (2008) propone que la teoría de la desregulación emocional es un proceso que tiene varios componentes, dos de ellos son los más significativos, el de la valoración cognoscitiva y el de la eliminación de la manifestación emocional (Porro, Andrés y Rodríguez-Espínola, 2012). La regulación emocional es, entonces, un proceso por el cual la persona evalúa un evento con una carga emocional alta, a partir de las vivencias y la estructuración de los esquemas cognitivos que forman los mismos. En cuanto a la expresión emocional, existen investigaciones que la han evaluado desde la regulación del grupo de emociones y sentimientos. De ahí que algunos han considerado que más que una revaluación de los hechos, como lo piensa Gross (1999), tiene que ver con el pensamiento analítico (Medrano, Muñoz y Cano, 2016).

Otros especialistas en el tema abordan la variable de la desregulación emocional en la adolescencia, momento crítico del desarrollo en el que la persona pone en juego el cúmulo de aprendizajes de la infancia en su normalización dentro del conjunto de la red social. Allí la regulación es una herramienta neurobiológica y psicológica que posibilita calidad de vida mediante la adaptación (Gresham y Gullone, 2012; Vera, Rodríguez, Tánori y Grubits, 2018).

A partir del estudio de la desregulación emocional, se han elaborado instrumentos psicológicos, entre los que se destacan la Escala para la Estimación de las Dificultades de Regulación Emocional (DERS - Versión en español). Revisiones al DERS han permitido que se extienda a personas de habla hispana. Por medio de este instrumento y la teoría expuesta de manera sucinta, se han hallado índices sobre la relación entre la desregulación emocional y la conducta antisocial (Gómez-Simón, 2015; Guzmán-González, Trabucco, Urzúa y Leiva, 2014; Medrano y Trólogo, 2014). En poblaciones de Latinoamérica, la aplicación de este instrumento ha encontrado garantía de confiabilidad y validez; por ello, se puede utilizar para medir las dificultades de regulación emocional en el contexto colombiano (Chaplo, Kerig, Modrowski y Bennet, 2017; Christian, Meltzer, Thede y Kosson, 2017; ContrerasValdez, Hernández-Guzmán y Freyre, 2018; Wang, Lei, Yang, Gao y Zhao, 2017). Este instrumento fue construido a partir de las variables que se entienden como facilitadoras de la regulación autonómica 
106 GERMÁN CABRERA GUTIÉRREZ, NORA HELENA LONDOÑO ARREDONDO, JOAN SEBASTIÁN ARBELÁEZ CARO, JUAN DAVID CRUZ VALENCIA, LEIDY YOVANNA MACÍAS CASTILLO Y ANA MARÍA ESPAÑA MACÍAS

del sujeto: rechazo, descontrol, interferencia, desatención y confusión emocional.

Por su parte, Muñoz-Martínez, Vargas y HoyosGonzález (2016) describen las mencionadas variables como dominio; regulación emocional, que implica la conciencia de la emoción; y desregulación, que supone una pérdida del conocimiento y razonamiento lógico sobre la misma, es decir, atender y entender. Para GuzmánGonzález et al. (2014), el dominio de las estrategias emocionales es una herramienta que surge del control emocional, pues el sujeto entiende la emoción y la usa para su adaptación. En tanto que Muñoz-Martínez et al. (2016) definen el dominio de la claridad en consonancia con las dos anteriores, y supone experimentar la emoción en la resolución de problemas, lo que también requiere de la conciencia subjetiva de la consecuencia de no tener claridad de la emoción. Gómez-Simón (2015) se refiere al dominio de los impulsos desde una óptica biológica, al argüir que la desregulación emocional es un aspecto ejecutivo del procesamiento cerebral. Según este dominio, el sujeto puede inhibir la emoción que es desadaptativa para su mejor uso, lo que va de la mano con el dominio de las metas por las cuales el sujeto puede realizar el proceso empático de anticipación y así elaborar estrategias y correcciones de aspectos negativos.

Así las cosas, la desregulación de las emociones es una variable de riesgo que permite la aparición de comportamiento antisocial. De ahí que los adolescentes sean un grupo especialmente vulnerable (Alarcón, Pérez-Luco, Wenger, Salvo y Chesta, 2018; Martínez-Vilchis, Morales y Pozas, 2018), debido al momento crítico que atraviesan de su ciclo vital. Investigaciones actuales en el tema han comprobado estas hipótesis sumando también elementos como el origen étnico y la relación de la desregulación emocional con la capacidad de frustración (Novin, Bos, Stevenson y Rieffe, 2017) o el género (Riley, 2018). Otros han confirmado la conjetura teórica del modelo epigenético, ya que el aprendizaje social media en la aparición de comportamiento antisocial, en tanto este es modulado por la capacidad de regulación emocional (Gillespie, Brzozowski y Mitchell, 2017; Weissman et al., 2017).

La vulnerabilidad en la adolescencia está dada por las correspondencias entre los aspectos biológicos y de ajuste social del individuo. En psicología, sin duda uno de los teóricos más importantes acerca de la adolescencia es Hall, quien publicó en 1904 un estudio científico sobre la adolescencia y fue el precedente de la posición epigenética de Erikson. Sin embargo, Hall pone atención primeramente en los factores genéticos y los cambios en el adolescente, los cuales son propios de la pubertad, poniendo a la socialización y a la cultura en un segundo renglón. También inaugura para la psicología la comprensión de la adolescencia, en términos de lo que él mismo denomina un periodo de tormenta y estrés. Esto significa que, como el foco de atención es la pubertad y sus factores biológicos asociados, entonces se considera que estos también son las causas de los cambios físicos y psicológicos, además de los inter e intrarrelacionales.

La tormenta y el estrés que se observan en la época de la adolescencia, producto de la pubertad, son más que solo épocas problemáticas, constituyen un periodo de renovación y afianzamiento del sujeto. La posición cognitiva-constructivista, dentro de la psicología, propone al adolescente como el sujeto que está en el periodo de afianzamiento de los esquemas, el cual abarca una serie de comportamientos experimentales en los que se definen la independencia y la exploración de habilidades y aptitudes diferenciales. Aquí es posible hablar de personalidad y de factores como los rasgos del sujeto. Según Lozano (2014), "la adolescencia se define como un periodo de transición en el estatus biosocial del individuo: el periodo que transcurre entre la madurez biológica y social" (p. 28).

La afectividad y la regulación emocional, así como el seguimiento de normas, están contenidos dentro del desarrollo cognitivo en su génesis, pero tienen expresiones en el desarrollo social y físico. Sin embargo, aunque la teoría ha explicado el desarrollo cognitivo y afectivo en el adolescente, investigaciones en Colombia han demostrado que muchos de los adolescentes en edades escolares no alcanzan etapas del pensamiento formal. Al respecto, Henao y Solórzano (2012) recopilaron investigaciones que identificaron el nivel de juicio y de pensamiento de adolescentes colombianos a la luz de las teorías piagetianas, encontrando que los adolescentes pueden realizar operaciones formales sin llegar a deducciones hipotéticas y deductivas, y concluyen que "la mayoría de nuestros adolescentes colombianos se encuentran en la fase inicial del 
pensamiento formal" (De Zubiría y De Zubiría citados en Henao y Solórzano, 2012, p. 59). No obstante, dichas investigaciones deben actualizarse y plantearse frente a nuevas y renovadas teorías, además de efectuar un análisis atendiendo a las necesidades particulares del medio colombiano. Por su parte, Velásquez (2007) recopiló algunos aspectos o categorías que identifican el lenguaje e identidad de los adolescentes en el contexto colombiano, refiriendo cinco condiciones: (a) pertenencia a tribus, (b) corporeísmo, (c) emocionalidad exaltada, (d) presentismo y (e) uso particular del lenguaje. Estos elementos pueden constituirse como factores de riesgo, configurando así la condición de vulnerabilidad en los adolescentes.

Las conductas antisociales y delictivas son, entonces, un factor de riesgo importante para los adolescentes en Colombia, pues existe una alta prevalencia de estas conductas en dicha población. Según datos del Instituto Colombiano de Bienestar Familiar (ICBF), para la fecha de corte en junio 30 de 2018, se encontraban en el Sistema de Responsabilidad Penal para Adolescentes (SRPA) 251.455 menores. De estos, la mayoría (92.736) tenían 17 años, seguidos de jóvenes de 16 años (76.991). El delito que más se comete, según el ICBF (2018), es el hurto, con el 36.32\%, seguido del tráfico, fabricación o porte de estupefacientes, con el $26.81 \%$.

En este sentido, la presente investigación versa sobre los factores implicados en la conducta antisocial, específicamente sobre la desregulación emocional en adolescentes que están en contextos del SRPA. El propósito fue identificar la relación entre la desregulación emocional y la conducta antisocial y delictiva en adolescentes que se encuentran vinculados a sistemas de responsabilidad penal, además de comprobar si existen diferencias en la regulación emocional de adolescentes con y sin problemas de conducta antisocial.

Asimismo, se identificaron factores asociados con la conducta antisocial, que afecta de forma negativa el orden social en el contexto del departamento del Quindío, Colombia. Con esta revisión, y una vez conocidos algunos de estos factores, se buscó promover o generar, a través de la intervención de las entidades gubernamentales y de otro orden, planes de acción y de fortalecimiento institucional, social y comunitario para el desarrollo de mejores condiciones familiares e individuales, en el proceso de formación en habilidades de regulación emocional para los niños, niñas y adolescentes de forma preventiva y promocional, como aporte a la disminución de la conducta antisocial en adolescentes y su posterior vinculación con el SRPA diseñado por el Estado para la sanción de los mismos.

\section{Método}

\section{Diseño}

La investigación tuvo un enfoque cuantitativo, no experimental, con un alcance descriptivo, correlacional y de temporalidad transversal, con la que se buscó obtener información acerca del grado de relación entre las variables, en este caso, entre la desregulación emocional y la conducta antisocial.

\section{Muestra}

Se propuso como población general, los adolescentes y jóvenes pertenecientes al SRPA en Colombia. Puntualmente, el universo muestral correspondió a los adolescentes vinculados al SRPA del departamento del Quindío en 2018, que corresponden a 246. Para la elección de la muestra se tuvo en cuenta un método no probabilístico de tipo intencional. Al final, la muestra estuvo compuesta por un total de 62 sujetos. En la selección se consideraron los siguientes criterios de inclusión: (a) la firma del consentimiento informado por parte de los adultos responsables y de los adolescentes; y (b) un rango de edad entre 14 y 18 años. Como criterios de exclusión se tuvieron en cuenta los trastornos mentales de base y el deterioro cognitivo.

Además, la muestra original se redujo por las siguientes características: (a) la dificultad para que los padres de familia consintieran el estudio, debido a que se debía esperar a las reuniones o visitas de familias a las instituciones, lo cual dependía de la lejanía de los padres de las distintas instituciones; y (b) el consumo de sustancias al momento de responder.

\section{Instrumentos}

Entre los instrumentos utilizados se encuentra, en primer lugar, la Entrevista Neuropsiquiátrica para Niños y Adolescentes (MINI KID) en su versión en español de Colón-Soto, Díaz, Soto y Santana (2005). 
108 GERMÁN CABRERA GUTIÉRREZ, NORA HELENA LONDOÑO ARREDONDO, JOAN SEBASTIÁN ARBELÁEZ CARO, JUAN DAVID CRUZ VALENCIA, LEIDY YOVANNA MACÍAS CASTILLO Y ANA MARÍA ESPAÑA MACÍAS

Se aplicaron los ítems L (dependencia y abuso de alcohol), M (dependencia y abuso de sustancias), $\mathrm{P}$ (trastornos de la conducta disocial) y R (trastorno psicótico), los cuales se eligieron de la prueba, dado que hay una problemática alta de consumo en la población objeto y, por otro lado, el trastorno de conducta se relaciona, pues en muchos casos está implícita y se asocia con trastornos psicóticos.

El segundo instrumento fue la Escala de Desregulación Emocional (DERS-E; Hervás y Jódar, 2008), la cual tiene por objeto observar la capacidad que tiene un sujeto de reanudar su estabilidad emocional cuando ha sido alterada, lo que implica una serie de componentes psicológicos y específicamente la valencia emocional que poseen. Los elementos utilizados como escalas generales o dominios en la desregulación emocional fueron: conciencia, claridad, impulso, estrategias, metas y no aceptación. La DERS-E, en su versión original adaptada al español, según Hervás y Jódar (2008), consiste en 5 dominios: descontrol, rechazo, interferencia, desatención y confusión. Cada una de las dimensiones tenía una alta confiabilidad en el alfa de Cronbach, que osciló entre 0.87 y 0.94 . Además, estaba confirmada por una investigación realizada por Marín, Robles, González-Forteza y Andrade (2012).

En una muestra aplicada en Chile, los autores decidieron realizar la prueba según el modelo español, encontrando una alta confiabilidad que en total marcó un alfa de 0.92. En el caso de Colombia, Muñoz-Martínez et al. (2016) reportaron solidez estadística en las dimensiones conciencia y estrategias. En la presente investigación, la escala mostró una alta confiabilidad, que osciló entre 0.89 y 0.90 . Cabe resaltar que la escala DERS-E mide la capacidad del sujeto para no aceptar las respuestas emocionales, con lo que se quiere dar un conocimiento global de la emoción, las estrategias y los baches, y si se está en la capacidad de reconocer el componente emocional como tal.

Se empleó también una ficha de caracterización con la que se indagó por las características psicosociales de los adolescentes encuestados, haciendo énfasis en la familia.

\section{Consideraciones éticas}

La presente investigación contó con el aval del Comité de Investigaciones y Bioético de la
Universidad de San Buenaventura Medellín. Además, el ejercicio de investigación se llevó a cabo siguiendo las recomendaciones dispuestas en la Ley 1090 de 2016, específicamente en su capítulo VII. Se orientó a los participantes sobre las consideraciones éticas propias: (a) secreto y buen manejo de la información, (b) derecho a la no participación, (c) derecho a la información y al acompañamiento y $(\mathrm{d})$ respeto por la intimidad.

\section{Procedimiento}

Una vez delimitada la teoría y los instrumentos para la recolección de los datos, se explicaron las condiciones éticas de la investigación a los adolescentes y padres; luego se procedió con la aplicación de las encuestas en las instituciones, centros de internamiento preventivo, centros de atención especializada y centros en modalidad de internado, semicerrado y externado que fueran contratados por el ICBF regional Quindío, en un periodo de 12 meses (de diciembre de 2017 a diciembre de 2018). Tras aplicar los instrumentos, se continuó con su análisis estadístico gracias al software estadístico SPSS (versión 23). El plan de análisis constó de dos partes: por un lado, el análisis descriptivo de la población, y luego, el análisis bivariado, siguiendo la línea de la estadística no paramétrica, dadas las características de las variables de estudio. Para el caso del análisis univariante, se realizaron pruebas de hipótesis KolmogorovSmirnov, con lo que se estableció la no normalidad. Posterior a ello, se aplicó el estadístico T-Student para comparar los grupos. Por último, para indicar la influencia de la desregulación emocional con la conducta antisocial, se utilizó una regresión lineal múltiple, cumpliendo con los supuestos de no multicolinealidad, independencia de observaciones y linealidad (prueba de Durbin-Watson).

\section{Resultados}

La muestra estuvo compuesta por 62 adolescentes. La edad promedio era de 16 años $(D E=0.87)$ y un estrato socioeconómico promedio de 2 . En cuanto a la escolaridad, el último grado alcanzado era octavo de secundaria, en promedio. Los adolescentes encuestados llevaban cinco meses, en promedio, en las instituciones del SRPA. En las tablas 1 y 2 se sintetizan los factores descriptivos de la población. 
Tabla 1

Factores personales y familiares

\begin{tabular}{|c|c|c|c|}
\hline & & $n$ & $\%$ \\
\hline \multirow{2}{*}{ ¿Cuál es su género? } & Hombre & 56 & 90.3 \\
\hline & Mujer & 6 & 9.7 \\
\hline \multirow{10}{*}{ ¿Cuál es la ciudad de residencia? } & Armenia & 41 & 66.1 \\
\hline & Montenegro & 6 & 9.7 \\
\hline & Quimbaya & 1 & 1.6 \\
\hline & Calarcá & 4 & 6.5 \\
\hline & Cali & 1 & 1.6 \\
\hline & La Tebaida & 5 & 8. \\
\hline & Caicedonia & 1 & 1.6 \\
\hline & Circasia & 1 & 1.6 \\
\hline & Córdoba & 1 & 1.6 \\
\hline & Pueblo Nuevo & 1 & 1.6 \\
\hline \multirow{3}{*}{ ¿Cuál es su estado civil? } & Soltero & 56 & 90.3 \\
\hline & Casado & 3 & 4.8 \\
\hline & Separado & 3 & 4.8 \\
\hline \multirow{3}{*}{$\begin{array}{l}\text { ¿Cuál era su ocupación antes del } \\
\text { SRPA? }\end{array}$} & Estudio & 39 & 62.9 \\
\hline & Trabajo & 9 & 14.5 \\
\hline & Ninguno & 14 & 22.6 \\
\hline \multirow{8}{*}{ ¿Cómo está constituida su familia? } & Familia nuclear conyugal & 2 & 3.2 \\
\hline & Familia nuclear de origen & 14 & 22.6 \\
\hline & Familia simultánea & 6 & 9.7 \\
\hline & Familia compuesta & 2 & 3.2 \\
\hline & Familia extendida & 7 & 11.3 \\
\hline & $\begin{array}{l}\text { Familia monoparental } \\
\text { masculina }\end{array}$ & 8 & 12.9 \\
\hline & $\begin{array}{l}\text { Familia monoparental } \\
\text { femenina }\end{array}$ & 21 & 33.9 \\
\hline & Grupos fraternos & 2 & 3.2 \\
\hline \multirow{13}{*}{ ¿Por cuál delito está en el SRPA? } & Homicidio & 4 & 6.5 \\
\hline & Hurto & 13 & 21.0 \\
\hline & Tráfico de estupefacientes & 12 & 19.4 \\
\hline & Concierto para delinquir & 3 & 4.8 \\
\hline & Abuso sexual & 2 & 3.2 \\
\hline & $\begin{array}{l}\text { Violencia contra funcionario } \\
\text { público }\end{array}$ & 2 & 3.2 \\
\hline & Lesiones personales & 4 & 6.5 \\
\hline & Extorsión & 2 & 3.2 \\
\hline & Secuestro & 0 & 0.0 \\
\hline & Daño en bien ajeno & 1 & 1.6 \\
\hline & Violencia intrafamiliar & 11 & 17.7 \\
\hline & Tentativa de homicidio & 8 & 12.9 \\
\hline & Porte de armas & 0 & 0.0 \\
\hline
\end{tabular}

Fuente: elaboración propia. 
110 GERMÁN CABRERA GUTIÉRREZ, NORA HELENA LONDOÑO ARREDONDO, JOAN SEBASTIÁN ARBELÁEZ CARO, JUAN DAVID CRUZ VALENCIA, LEIDY YOVANNA MACÍAS CASTILLO Y ANA MARÍA ESPAÑA MACÍAS

El 54.8\% de la población presentaba problemas de conducta actual (conducta antisocial), frente al restante $45.2 \%$ que no lo presentaba. Al momento del estudio, la mayoría (62.9\%) de los participantes consumían marihuana, seguido de algún inhalante- estimulante $(8.1 \%)$, cocaína $(6.5 \%)$ y el $19.4 \%$ no consumían ninguna sustancia psicoactiva.

En la tabla 2 se presentan las medidas de tendencia central más importantes de la Escala de Desregulación Emocional.

Tabla 2

Medidas de tendencia central de la Escala de Desregulación Emocional

\begin{tabular}{lccccc}
\hline & $\begin{array}{c}\text { Rechazo } \\
\text { emocional }\end{array}$ & $\begin{array}{c}\text { Descontrol } \\
\text { emocional }\end{array}$ & $\begin{array}{c}\text { Interferencia } \\
\text { emocional }\end{array}$ & $\begin{array}{c}\text { Desatención } \\
\text { emocional }\end{array}$ & $\begin{array}{c}\text { Confusión } \\
\text { emocional }\end{array}$ \\
\hline$M$ & 16.42 & 21.31 & 10.82 & 14.06 & 10.27 \\
Mdn & 14.00 & 19.00 & 10.00 & 14.00 & 10.00 \\
DT & 8.432 & 9.415 & 4.860 & 3.772 & 3.179 \\
Mín. & 7 & 9 & 4 & 6 & 4 \\
Máx. & 35 & 42 & 20 & 20 & 17 \\
\hline
\end{tabular}

Fuente: elaboración propia.

Una vez realizada la descripción de los factores individuales y familiares, se pasó a indicar si existían o no diferencias respecto a la desregulación emocional en los grupos de adolescentes con y sin trastorno de conducta antisocial. Los resultados se presentan en la tabla 3.

Tabla 3

Diferencias entre los factores de la desregulación emocional y las poblaciones de estudio

\begin{tabular}{lcc}
\hline DERS-E & $F$ & $p$ \\
\hline Rechazo emocional & 2.19 & 0.144 \\
Descontrol emocional & 2.96 & 0.091 \\
Interferencia emocional & 0.14 & 0.711 \\
Desatención emocional & 4.86 & 0.031 \\
Confusión emocional & 0.30 & 0.589 \\
\hline
\end{tabular}

Fuente: elaboración propia.

Tal como se indica en la tabla 3, la única variable que representa una diferencia en la población de adolescentes con y sin problema de conducta antisocial es la desatención emocional.

Por último, para indicar la probabilidad de la aparición de conducta antisocial en los adolescentes de la muestra, se realizó una regresión logística binaria a partir de la variable dependiente, trastorno disocial. Asimismo, se probaron tres modelos, y en el último se propusieron como variables independientes los factores de la desregulación emocional. El estadístico $R$ cuadrado de Nagelkerke, muestra que, con la entrada de la variable desregulación emocional, se explica en $72 \%$ la varianza del trastorno antisocial. Además, se encontró que la única variable que entra en la ecuación es la desatención emocional $(\beta=0.309$; $p=0.05 ; \operatorname{Exp}(\beta)=1.362$ ), lo que se traduce en que, a mayor desatención emocional, mayor probabilidad de que el adolescente presente síntomas asociados al trastorno disocial. 


\section{Discusión}

El propósito del estudio fue establecer el grado de relación entre la desregulación emocional y la conducta antisocial y delictiva en adolescentes. Como primer hallazgo significativo, se observó que las variables sobre la regulación emocional que puntuaron por encima de lo esperado, fueron la desatención de las emociones y la interferencia emocional. Sin embargo, los datos no son significativamente altos, pero ilustran y corroboran, como se ha observado en otras investigaciones (González, Souto y Fernández, 2017; López et al., 2017), que la atención de las emociones se asocia, en general, con problemas de regulación emocional. Para el presente caso, aunque tampoco se relaciona estadísticamente con la conducta antisocial, la atención a las propias emociones y la interferencia son indicadores lejanos de problemáticas conductuales. Otras investigaciones (Amézquita, 2017) no encontraron relación entre estas subescalas y la regulación emocional.

Por otro lado, de acuerdo con los hallazgos, fue posible comprobar, siguiendo la línea de Gross (1999), que la emoción implica un estímulo del cual se deriva una serie de respuestas informacionales, entre las cuales, el aspecto conductual es el más significativo, pues retroalimenta el comportamiento desadaptativo de la persona, evidenciando patrones de adaptación socialmente inadecuados (Balaguer, Sánchez y García, 2018). En la misma línea, Gioia (citada en Ramos, Bolaños, Paredes y Ramos, 2016) construye un modelo de comprensión de la regulación emocional, entendiéndola como una función ejecutiva, ya que está implicada en los procesos metacognitivos del sujeto, lo que confirma aún más el trabajo de Gross.

Una de las hipótesis en las que reposa el presente estudio, es que hay una relación de probabilidad en la aparición de conductas antisociales y la desregulación emocional. Se comprobó, mediante un análisis de regresión, que la desatención emocional es el factor de la regulación emocional que predice dicha conducta de riesgo. Asimismo, que es el de mayor presencia en los grupos de estudio, lo que corrobora la importancia de esta variable específica en los diferentes modelos clínicos de atención en salud mental. La evidencia mostró que la desatención está involucrada en la vulnerabilidad a sufrir síntomas transdiagnósticos presentes en gran cantidad de patologías, como lo es el caso de las patologías duales (García-Pardo, 2018) o depresión clínica asociada con trastornos neurocognitivos (Da Silva, Carvalho y Ramos, 2019). En suma, la desatención emocional funciona como un tipo de esquema con el que el sujeto evalúa de manera negativa las experiencias y su capacidad de autogestión. Igualmente, con el comportamiento antisocial en adolescentes, la desatención emocional favorece el comportamiento delictivo, al permitir el alejamiento de la responsabilidad legal y moral del sujeto, el cual se da en cierta forma por la gestión adecuada de emociones y sentimientos (Rodríguez, Herrera y Rodríguez, 2018).

El aspecto emocional está relacionado con problemas de conducta y también en la conducta antisocial. Así, fue posible demostrar la hipótesis principal, la cual indicaba que quienes muestran problemas de conducta, poseen problemas en la regulación de sus emociones $\mathrm{o}$, al menos, que existe un indicador de tal relación. Se evidenció que el $54.8 \%$ de los adolescentes presentaron trastorno de conducta y que este no se relacionaba con la desregulación de las emociones. Sin embargo, el Manual diagnóstico y estadístico de los trastornos mentales (DSM versión V), de la American Psychiatric Association (APA, 2014), considera que el trastorno de conducta tiene como principal factor la negación del entorno del sujeto, sobre la que hay una respuesta de rechazo y agresión. En este entorno se encuentran los iguales, figuras de autoridad y la norma. No todas las personas que están en el SRPA manifiestan este trastorno y tampoco todos presentan desregulación emocional, lo que contraría la evidencia de otras investigaciones al respecto (Côté et al., 2017; Estévez y Jiménez, 2017; Loeber, Farrington y Redondo, 2011; Pérez, 2017).

Como se expuso anteriormente, el estrato socioeconómico promedio de los participantes del ejercicio es 2, lo que refleja un bajo nivel en cuanto al acceso a recursos que provean normales y esperados indicadores de calidad de vida. Aunque tampoco se halló relación entre la conducta antisocial y la desregulación con respecto al estrato socioeconómico, el contexto social en el que se despliega la familia, como el lugar de desarrollo del sujeto, influye especialmente en la regulación emocional. Así lo ilustran Lalayants y Prince (2014), quienes afirman que podría existir una especie de 
112 GERMÁN CABRERA GUTIÉRREZ, NORA HELENA LONDOÑO ARREDONDO, JOAN SEBASTIÁN ARBELÁEZ CARO, JUAN DAVID CRUZ VALENCIA, LEIDY YOVANNA MACÍAS CASTILLO Y ANA MARÍA ESPAÑA MACÍAS

circularidad entre diversos elementos que incluyen el hecho de una madre que posee problemas de conducta o consumo de sustancias, aspecto que es el más preocupante, pues la tasa de consumo en el departamento del Quindío, lugar de procedencia de la mayoría de los adolescentes encuestados, es muy alta. De hecho, para 2017, la prevalencia del consumo de marihuana era del $6.34 \%$ y la de cualquier sustancia, del $6.8 \%$, siendo la segunda más alta en Colombia. De esta manera, familia, consumo y contexto inciden determinantemente en los adolescentes para la aparición de desregulación emocional, problemas de conducta y conducta antisocial. Esto se afirma gracias a los datos encontrados entre las variables contextuales y las de análisis, lo que comprueba su relación en la teoría y la evidencia empírica (Ministerio de Justicia y del Derecho, 2017; Sánchez, Xóchilt y Robles, 2018).

Otro elemento para prestar atención fue que más del $90 \%$ de las personas solo habían ingresado una sola vez al SRPA, lo que muestra una baja tasa de reincidencia en el delito. Sin embargo, se podría explicar por el bajo nivel de desregulación emocional, ya que, como indica la evidencia (Wibbelink, Hoeve, Stams y Oort, 2017), hay una relación entre la posibilidad de reincidir en la conducta delictiva y los problemas de conducta. Esto, además, se orienta en una muestra que se toma de un sistema en el que existe una tasa de reincidencia de la conducta punible del $20.16 \%$ (ICBF, 2015). Así las cosas, para pensar en la no existencia de la reincidencia del delito, es importante no solo atender la regulación emocional en la familia como un factor de prevención, sino fortalecer el SRPA (Valencia, 2015), pensando en los datos encontrados y en los que la evidencia aporte, ya que, para comprender la conducta antisocial, es necesario estudiar la desregulación emocional y la familia con el contexto en el que se desenvuelve.

La principal limitante del estudio fue el reducido tamaño de la muestra. Se espera para próximas investigaciones ahondar en los factores asociados a la reincidencia del delito y la caracterización del contexto socioeconómico y familiar de los adolescentes indagados. Asimismo, dado que el muestreo fue no probabilístico, limitó los alcances del estudio, ya que este no permite control sobre la participación aleatoria de todos los sujetos que posiblemente podrían participar del estudio en todo el departamento del Quindío.
Otra limitación fue el hecho de que los datos sociodemográficos se obtuvieron a través de la entrevista con el participante y no mediante la revisión de otros documentos o entrevista con las familias. Esto indica la importancia de indagar por los factores familiares en la relación de la conducta antisocial y la regulación emocional (Andrés et al., 2017; Sánchez et al., 2018).

\section{Referencias}

Alarcón, P. A., Pérez-Luco, R. X., Wenger, L. S., Salvo, S. I. y Chesta, S. A. (2018). Personalidad y gravedad delictiva en adolescentes con conducta antisocial persistente. Revista Iberoamericana de Psicología y Salud, 9(1), 5874. doi.org/10.23923/j.rips.2018.01.015

Aldrete-Cortez, V., Carrillo-Mora, P., MansillaOlivares, A., Schnaas, L. y Esquivel, F. (2014). De la regulación emocional y cognitiva a la autorregulación en el primer año de vida. Anuario de Psicología, 44(2), 199-212.

American Psychological Association [APA]. (2014). Manual diagnóstico y estadístico de los trastornos mentales (5. ${ }^{a}$ ed.). México: Manual Moderno.

Amézquita, J. (2017). Estudio correlacional en estudiantes de noveno grado de dos instituciones educativas de la ciudad de Medellín (Tesis de maestría). Departamento de Psicología, Facultad de Ciencias Sociales y Humanas, Universidad de Antioquia, Medellín. Recuperado de http://bibliotecadigital.udea. edu.co/dspace/bitstream/10495/9664/1/ A m e zquita Ja i n e r_ 2017 _ RegulacionEmocionalAprovechamiento.pdf

Andrés, M. L., Stelzer, F., Vernucci, S., Canet, L., Galli, J. I. y Navarro, J. I. (2017). Regulación emocional y habilidades académicas: relación en niños de 9 a 11 años de edad. Suma Psicológica, 24(2), 79-86. doi.org/10.1016/j. sumpsi.2017.07.001

Balaguer, N., Sánchez, M. y García, A. (2018). Relación entre la regulación emocional y la autoestima. Àgora de Salut, 5(41), 373-380. doi.org/10.6035/AgoraSalut. 2018.5.41 
Carranza, J. (1994). Etología: Introducción a la ciencia del comportamiento. Cáceres: Universidad de Extremadura.

Chaplo, S. D., Kerig, P. K., Modrowski, C., \& Bennet, D. (2017). Gender Differences in the Associations Among Sexual Abuse, Posttraumatic Stress Symptoms, and Delinquent Behaviors in a Sample of Detained Adolescents. Journ Child Adol Trauma, 10, 29-39. doi: 10.1007/s40653016-0122-Z

Christian, E., Meltzer, C., Thede, L., \& Kosson, D. (2017). The Relationship Between Early Life Events, Parental Attachment, and Psychopathic Tendencies in Adolescent Detainees. Child Psychiatry Hum Dev, 48, 260-269. doi: 10.1007 / s10578-016-0638-3

Colombia. Ministerio de Justicia y del Derecho. (2017). Reporte de drogas de Colombia 2017. Bogotá, DC: Autor. Recuperado de http://www. odc.gov.co/Portals/1/publicaciones/pdf/odclibro-blanco/reporte_drogas_colombia_2017. pdf

Colón-Soto, M., Díaz, V., Soto, O. y Santana, C. (2005). Mini International Neuropsychiatric Interview para Niños y Adolescentes (MINIKID) Versión en español. Tampa: Medical Outcome Systems.

Contreras-Valdez, J., Hernández-Guzmán, L. y Freyre, M. (2018). Validez de constructo de la versión corta de la Escala de Desregulación Emocional para niños y adolescentes. Pensamiento Psicológico, 16(1), 19-31. doi:10.11144/Javerianacali.PPSI16-1.vcvc

Côté, S. M., Orri, M., Brendgen, M., Vitaro, F., Boivin, M., Japel, C.,... Tremblay, R. (2017). Psychometric properties of the Mental Health and Social Inadaptation Assessment for Adolescents (MIA) in a population-based simple. International Journal of Methods in Psychiatric Research, 26(4), 3-10. doi: 10.1002/ mpr. 1566

Da Silva, C. Y., Carvalho, P. A. y Ramos, V. (2019). La depresión y su influencia en los cambios neuropsicológicos del adulto mayor con trastorno neurocognitivo leve debido a la enfermedad de Alzheimer. Revista CES Psicología, 12(1), 69-79. doi: 10.21615/ cesp.12.1.6
Estévez, E. y Jiménez, T. (2017). Violencia en adolescentes y regulación emocional. International Journal of Developmental and Educational Psychology, 1, 97-104. doi: 10.17060/ijodaep.2017.n1.v2.922

Garaigordobil, M., Aliri, J., Martínez-Valderrey, V., Maganto, C., Bernaras, E. y Jaureguizar, J. (2013). Conducta antisocial: conexión con emociones positivas y variables predictoras. Apuntes de Psicología, 31, 123-133.

García-Pardo, A. (2018). El desarrollo de la estabilidad emocional en patología dual: una propuesta de intervención breve. Clínica y Salud, 29(3), 133-137. doi: 10.5093/ clysa2018a19

Gillespie, M., Brzozowski, A., \& Mitchell, I. (2017). Self-Regulation and Aggressive Antisocial Behaviour: Insights from Amygdala-Prefrontal and Heart-Brain Interactions. Psychology, Crime and Law, 24(3), 243-257. doi: 10.1080/1068316X.2017.1414816

Gómez-Simón, I. (2015). Dificultades específicas de la regulación emocional en adolescentes con TDAH: utilidad clínica de la escala DERS (Tesis de Doctorado en Psicología Clínica y de la Salud). Universidad Autónoma de Barcelona, España. Recuperado de https://core.ac.uk/ download/pdf/78535832.pdf

González, R., Souto, A. y Fernández, R. (2017). Perfiles de regulación emocional y estrés académico en estudiantes de fisioterapia. European Journal of Education and Psychology, 10(2), 57-67. doi: 10.1016/j.ejeps.2017.07.002

Gresham, D., \& Gullone, E. (2012). Emotion Regulation Strategy Use in Children and Adolescents: The Explanatory Roles of Personality and Attachment. Personality and Individual Differences, 52(5), 616-621. doi: 10.1016/j.paid.2011.12.016

Gross, J. (1999). Emotion and Emotion Regulation. In L. A. Pervin, \& O. P. John (Eds.), Handbook of Personality: Theory and research (2nd ed.). (pp. 525-552). New York: Guilford.

Gross, J. (2008). Emotion regulation. In M. Lewis, J. M. Haviland-Jones, \& L. F. Guilford (Eds.), Handbook of Emotions (3rd ed.). (pp. 497512). New York: G. In Press. 
114 GERMÁN CABRERA GUTIÉRREZ, NORA HELENA LONDOÑO ARREDONDO, JOAN SEBASTIÁN ARBELÁEZ CARO, JUAN DAVID CRUZ VALENCIA, LEIDY YOVANNA MACÍAS CASTILLO Y ANA MARÍA ESPAÑA MACÍAS

Gutiérrez, A., Herrero, D. y Zarabia, I. (2014). El papel mediador de la regulación emocional entre el juego patológico, uso abusivo de Internet y videojuegos y la sintomatología disfuncional en jóvenes y adolescentes. Adicciones, 26(4), 282-290. doi: 10.20882/ adicciones. 26

Guzmán-González, M., Trabucco, C., Urzúa, A., Garrido, L. y Leiva, J. (2014). Validez y confiabilidad de la versión adaptada al español de la Escala de Dificultades de Regulación Emocional (DERS-E) en población chilena. Terapia Psicológica, 32(1), 19-29. doi: 10.4067/ S0718-48082014000100002

Hall, G. S. (1904). Adolescence: Its psychology and its relations to physiology, anthropology, sociology, sex, crime, religion, and education. New York: D’Appleton \& Co.

Henao, M. y Solórzano, B. (2012). Una aproximación al desarrollo del pensamiento en el adolescente. Revista Universitaria Eafit, 31(100), 53-60.

Hervás, G. y Jódar, R. (2008). Adaptación al castellano de la Escala de Dificultades en la Regulación Emocional. Clínica y Salud, 19(2), 139-156.

Instituto Colombiano de Bienestar Familiar [ICBF]. (2015). Adolescentes jóvenes y delitos: elementos para la comprensión de la delincuencia en Colombia. Recuperado de: https://www.icbf.gov.co/sites/default/files/ delincuencia_juvenil_web.pdf

Lalayants, M., \& Prince, J. (2014). Delinquency, Depression, and Substance Use Disorder Among Child Welfare-Involved Adolescent Females. Child Abuse \& Neglect, 38(4), 797807. doi: 10.1016 / j.chiabu.2013.08.008

Loeber, R., Farrington, D. y Redondo, S. (2011). La transición desde la delincuencia juvenil a la delincuencia adulta. Revista Española de Investigación Criminológica, 1(9), 1-41.

López, M. (2008). Psicología de la delincuencia. España: Universidad de Salamanca.

López, D., Puddington, M., Jaramillo, M. A., Moiseff, C., Gagliesi, P. y Stoewsand, C. (2017). Evaluación de un programa de mindfulness. Efectos preliminares sobre regulación emocional y sintomatología. Psiencia, 9(3), 1-16. doi: 10.5872/psiencia/9.3.21

Lozano, A. (2014). Teoría de teorías sobre la adolescencia. Última Década, 40, 11-36.

Marín, M., Robles, R., González-Forteza, C. y Andrade, P. (2012). Propiedades psicométricas de la escala "Dificultades en la Regulación Emocional" en español (DERS-E) para adolescentes mexicanos. Salud Mental, 35(6), 521-526.

Martínez-Vilchis, R., Morales, T. y Pozas, J. (2018). Efectos de un programa de competencias emocionales en la prevención de cyberbullying en bachillerato. Pensamiento Psicológico, 16(1), 33-44. doi:10.11144/Javerianacali. PPSI16-1.epce

Medrano, R., Muñoz, R. y Cano, A. (2016). Procesos cognitivos y regulación emocional: aportes desde una aproximación psicoevolucionista. Ansiedad y Estrés, 22, 47-54. doi: 10.1016/j. anyes.2016.11.001

Medrano, L. y Trólogo, M. (2014). Validación de la Escala de Dificultades en la Regulación Emocional en la población universitaria de Córdoba, Argentina. Universitas Psychologica, 13(4), 1345-1356. doi:10.11144/Javeriana. UPSY13-4.vedr

Muñoz-Martínez, A., Vargas, R. y Hoyos-González, J. (2016). Escala de dificultades en regulación emocional (DERS): análisis factorial en una muestra colombiana. Acta Colombiana de Psicología, 19(1), 225-236. doi: 10.14718/ ACP.2016.19.1.10

Novin, S., Bos, M., Stevenson, C. E., \& Rieffe, C. (2017). Adolescents' Responses to Online Peer Conflict: How Self-Evaluation and Ethnicity Matter. Infant and Child Development, 27(2), e2067. doi: 10.1002/icd.2067

Pérez, S. (2017). Desarrollo socio-emocional de los menores infractores (Tesis de Maestría en Intervención e Investigación Socioeducativa). Facultad de Formación del Profesorado y Educación, Universidad de Oviedo, España. Recuperado de http://digibuo.uniovi.es/ dspace/bitstream/10651/43819/11/TFM_ SofiaMicaelaPerezSotomayor.pdf 
Porro, M., Andrés, M. y Rodríguez-Espínola, S. (2012). Regulación emocional y cáncer: utilización diferencial de la expresión y supresión emocional en pacientes oncológicos. Avances en Psicología Latinoamericana, 30(2), 341-355.

Ramos, C., Bolaños, M., Paredes, L. y Ramos, D. (2016). Tratamiento neuropsicológico del TDAH en preescolares: Entrenamiento de la función ejecutiva. Revista Ecuatoriana de Neurología, 25(1-3), 61-69.

Riley, T. (2018). Adolescent Emotion Expression, Emotion Regulation, and Decision-Making in Social Context (PhD thesis in Philosophy). Commonwealth University, Virginia. Recovered from https://scholarscompass.vcu.edu/cgi/ viewcontent.cgi ?article $=6584 \&$ context $=$ etd

Rodríguez, Y., Herrera, L. y Rodríguez, G. (2018). Comparación de la prosocialidad en adolescentes con dificultades para aprender. Humanidades Médicas, 18(2), 258-272.

Sánchez, A., Xóchilt, I. y Robles, F. (2018). Conductas antisociales-delictivas en adolescentes: relación con el género, la estructura familiar y el rendimiento académico. Alternativas en Psicología (38), 80-98.

Valencia, J. (2015). La resocialización y la reincidencia de adolescentes en conductas delictivas en el departamento de Caldas, Colombia. Summa luris, 3(2), 377-390. doi: $10.21501 / 23394536.1834$
Velásquez, A. (2007). Lenguaje e identidad en los adolescentes de hoy. El Ágora USB, 7(1), 183197.

Vera, J., Rodríguez, C., Tánori, J. y Grubits, H. (2018). Recursos de ajuste psicosocial y su relación con la satisfacción con la vida en jóvenes de México. Pensamiento Psicológico, 16(2), 8797. doi: 10.11144/Javerianacali.PPSI16-2.rapr

Wang, X., Lei, L., Yang, J., Gao, L., \& Zhao, F. (2017). Moral Disengagement as Mediator and Moderator of the Relation Between Empathy and Aggression Among Chinese Male Juvenile Delinquents. Child Psychiatry, 48(2), 316-326. doi: 10.1007/s10578-016-0643-6

Weissman, D. G., Gelardi, K. L., Conger, R. D., Robins, R. W., Hastings, P. D., \& Guyer, A. E. (2017). Adolescent Externalizing Problems: Contributions of Community Crime Exposure and Neural Function During Emotion Introspection in Mexican-Origin Youth. Journal of Research on Adolescence: The Official Journal of the Society for Research on Adolescence, 28(2), 551-563. doi: 10.1111/ jora. 12358

Wibbelink, C., Hoeve, M., Stams, G., \& Oort, F. (2017). A Meta-Analysis of the Association between Mental Disorders and Juvenile Recidivism. Aggression and Violent Behavior, 33, 78-90. doi: 10.1016/j.avb.2017.01.005

Para citar este artículo / To cite this article / Para citar este artigo: Cabrera, G., Londoño, N., Arbeláez, J., Cruz, J., Macías, L. y España, A. (2020). Desregulación emocional y conducta disocial en una muestra de adolescentes en conflicto con la ley. Pensamiento Psicológico, 18(1), 103-115. doi:10.11144/Javerianacali.PPSI18-1.decd 